\title{
Mechanical Circulatory Support
}

National Cancer Institute

\section{Source}

National Cancer Institute. Mechanical Circulatory Support. NCI Thesaurus. Code C99540.

Use of a device to replace the function of a cardiac ventricle. This includes use of cardiopulmonary bypass, left ventricular assist device (LVAD), intra-aortic balloon pump (IABP) and/or extracorporeal membrane oxygenation (ECMO). 